\title{
Quantitative Determination of Hydroxymethanesulfonate (HMS) Using UHPLC-LTQ-Orbitrap Mass Spectrometry: A Missing Sulfur Source during Haze Episodes in Beijing
}

\author{
LIANFANG WEI ${ }^{1}$
}

1 State Key Laboratory of Atmospheric Boundary Layer Physics and Atmospheric

Chemistry, Institute of Atmospheric Physics, Chinese Academy of Sciences, Beijing

100029, China (weilianfang@mail.iap.ac.cn)

\begin{abstract}
Frequently occurred severe haze events in heavily populated China in recent years, especially in winter. Sulfate, as the major inorganic component of fine particles $\left(\mathrm{PM}_{2.5}\right)$, has been found to form rapidly under high relative humidity conditions. Currently, a large gap exists in model-estimated sulfate concentration and field observation. The hydroxymethanesulfonate (HMS) chemistry in the aerosol liquid water and fog/cloud droplet had been pointed to a potential missing sulfur-containing compounds during haze episodes. HMS is known to be formed during the reaction of $\mathrm{S}(\mathrm{IV})\left(\mathrm{HSO}_{3}{ }^{-}\right.$or $\left.\mathrm{SO}_{3}{ }^{2-}\right)$ with formaldehyde in the aqueous phase. And it can act as a reservoir species for S(IV) in the atmosphere and may be misidentified as sulfate using Ion Chromatography (IC) method. It is therefore of interest for the understanding of atmospheric sulfur chemistry.

Here we present an analytical technique for quantification of HMS components in aerosol using UHPLC-LTQ-Orbitrap Mass Spectrometry. The determination was carried out by its retention time and negative ion peak at $\mathrm{m} / \mathrm{z}-111$. The HMS components in $\mathrm{PM}_{2.5}$ samples collected in wintertime 2015 in Beijing were analyzed. HMS mass concentrations in the samples were highly variable, ranging from below the detection limit to around $7.0 \mu \mathrm{g} \mathrm{m}^{-3}$. Higher concentrations were associated with occurrence of fog events, which is the presumed source of the HMS. It suggested that HMS can be a potentially effective tracer of enhanced aqueous sulfur chemistry during the haze-fog episodes.
\end{abstract}

